Research Article

\title{
Propranolol Is Associated with Lower Risk of Incidence of Hepatocellular Carcinoma in Patients with Alcoholic Cirrhosis: A Tertiary-Center Study and Indirect Comparison with Meta-Analysis
}

\author{
Tzu-Hao Li $\mathbb{D}^{1,2,3}$ Yu-Lien Tsai, ${ }^{3,4}$ Chien-Fu Hsu, ${ }^{3,4}$ Chih-Wei Liu, ${ }^{2,3,5}$ \\ Chia-Chang Huang, ${ }^{2,3,6}$ Ying-Ying Yang $\mathbb{D}^{2,3,6,7}$ Hung-Cheng Tsai, ${ }^{3,5}$ Shiang-Fen Huang, ${ }^{2,3,4}$ \\ Yun-Cheng Hsieh, ${ }^{3,7}$ Hsuan-Miao Liu, ${ }^{8}$ Tzung-Yan Lee, ${ }^{8}$ Ming-Chih Hou, ${ }^{3,7}$ \\ Chang-Youh Tsai, ${ }^{3,5}$ and Han-Chieh Lin (iD ${ }^{3,7}$
}

${ }^{1}$ Division of Allergy, Immunology, And Rheumatology, Department of Internal Medicine, Shin Kong Wu Ho-Su Memorial Hospital, No. 95, Wen Chang Rd., Shihlin District, Taipei 111, Taiwan

${ }^{2}$ Institute of Clinical Medicine, National Yang-Ming University, No. 155, Sec. 2, Linong St., Beitou District, Taipei City 112, Taiwan

${ }^{3}$ Faculty of Medicine, National Yang-Ming University, No. 155, Sec. 2, Linong Street, Beitou District, Taipei City 112, Taiwan

${ }^{4}$ Department of Medicine, Taipei Veterans General Hospital, No. 201, Sec. 2, Shipai Rd., Beitou District, Taipei City 112, Taiwan

${ }^{5}$ Division of Allergy, Immunology, And Rheumatology, Department of Medicine, Taipei Veterans General Hospital, No. 201, Sec. 2, Shipai Rd., Beitou District, Taipei City 112, Taiwan

${ }^{6}$ Division of Clinical Skills Training, Department of Medical Education, Taipei Veterans General Hospital, No. 201, Sec. 2, Shipai Rd., Beitou District, Taipei City 112, Taiwan

${ }^{7}$ Division of Gastroenterology, Department of Medicine, Taipei Veterans General Hospital, No. 201, Sec. 2, Shipai Rd., Beitou District, Taipei City 112, Taiwan

${ }^{8}$ Graduate Institute of Traditional Chinese Medicine, Chang Gung University, No. 259, Wenhua 1st Rd., Guishan Dist., Taoyuan City 333, Taiwan

Correspondence should be addressed to Ying-Ying Yang; yangyy@vghtpe.gov.tw

Received 9 November 2019; Accepted 20 March 2020; Published 9 April 2020

Academic Editor: Oronzo Brunetti

Copyright (C) 2020 Tzu-Hao Li et al. This is an open access article distributed under the Creative Commons Attribution License, which permits unrestricted use, distribution, and reproduction in any medium, provided the original work is properly cited.

Alcoholic cirrhosis (AC) leads to enormous disease burden and occupies a substantial proportion in the etiology of hepatocellular carcinoma (HCC), but scarce attention has been paid to this topic. Besides, propranolol has been reported to decrease the rate of HCC in viral hepatitis. We conducted a retrospective tertiary-center cohort study to identify the HCC incidence in AC patients with or without propranolol. A total of 1,046 AC patients with hospitalization had been screened, and those with regular followup for three years or otherwise until the date of malignancy diagnosis without meeting exclusion criteria were enrolled; finally, 23 AC patients with propranolol and $46 \mathrm{AC}$ patients without propranolol were analyzed after twofold propensity-score matching. The cumulative incidence of HCC was lower in the propranolol group (log-rank test, $P=0.046$ ). Furthermore, we undertook the meta-analysis of annual incidence of HCC in AC patients, and 1,949 publications were screened, within which eight studies were analyzed; the pooled annual incidence was $2.41 \%$, which was higher than the calculated annual incidence of HCC in our AC cohort with propranolol (1.45\%). In conclusion, propranolol is associated with decreased risk of HCC incidence in patients with AC. 


\section{Introduction}

Cirrhosis is characterized by various complications, including high risk of developing hepatocellular carcinoma (HCC), and the necessity of policies regarding HCC surveillance has been proposed [1]. According to the costeffectiveness benefit, an incidence of HCC of at least $1.5 \%$ per year should undergo HCC screening [2]. Although a major epidemiological study estimated that approximately $30 \%$ of new primary liver cancer cases could be ascribed to alcohol, the annual incidence of HCC in alcoholic cirrhosis (AC) had been reported as a wide range from 0.4 to $5.6 \%$ [3-5]; therefore, a more comprehensive investigation should be performed.

Owing to the high risk of HCC in AC, seeking effective medication for HCC prevention is essential. A nonselective beta blocker (NSBB), propranolol, has been proven to have pleiotropic benefits for patients with cirrhosis, including being able to correct portal hypertension, prevent variceal bleeding, reduce the incidence of ascites, and decrease the risk of cirrhotic decompensation; therefore, propranolol is widely used in cirrhotic patients $[6,7]$. On the other hand, in the case other than HCC, propranolol possesses antitumor entities in several types of tumor [8-11]; however, there has been scarce literature regarding the influences of propranolol on HCC incidence in AC patients.

As was pointed out above, here in the present study, we undertook a retrospective propensity-score matching (PSM) analysis to investigate whether long-term propranolol treatment had protective effects on the incidence of HCC; furthermore, we conducted a meta-analysis to retrieve the pooled annual incidence of HCC in AC patients for integrative recognition, and comparison of the effects of long-term propranolol use.

\section{Methods}

2.1. Subjects. We conducted a retrospective tertiary-center cohort study, of which the protocol was approved by the Institutional Review Board of Taipei Veterans General Hospital. The medical and hospitalization records of AC subjects older than 18 years within the years 2006 and 2017 were reviewed. For the propranolol group, we enrolled subjects who had been hospitalized due to AC and regularly treated with propranolol for three consecutive years or otherwise until the date of HCC diagnosis; for the control (nonpropranolol) group, we analyzed the $\mathrm{AC}$ patients who regularly follow up without propranolol treatment. The criteria of exclusion consisted of patients younger than 18, treatment with hepatobiliary surgery or liver transplantation, history of primary liver cancer (HCC or cholangiocarcinoma) or malignancies before enrolment, and the initiation of propranolol use before the year 2006. The follow-up duration of each subject was three years or otherwise until the date of HCC diagnosis according to the available medical records, and those with new-onset malignancy other than HCC or loss of follow-up were censored.

The basic demographic data, including the age, sex, superimposed chronic liver disease, date of cirrhosis diagno- sis, initiation date of propranolol, and Child-Pugh scores, were acquired. Comorbidities including hypertension, hyperlipidemia, ischemic heart disease, chronic obstructive pulmonary disease, diabetes mellitus (DM), tuberculosis, and endstage renal disease were also recorded.

2.2. Outcome Measurement. The primary outcome in our study was the incidence of HCC, which was defined according to diagnostic image presentation or tissue proof, along with the corresponding catastrophic illness certificate. As to the catastrophic illness certificate of national health insurance, the medical records and diagnoses were reviewed by independent subspecialists to ensure the accuracy of diagnosis. The diagnosis of malignancy other than HCC and mortality was defined as a secondary endpoint.

2.3. Literature Review and Meta-Analysis. In comparison with the previous literature, we identified the relevant studies written in English or Chinese, focusing on the incidence of HCC in AC, from the PubMed, EMBASE, Airiti Library, and Cochrane Central Register of Controlled Trials (CENTRAL) databases, which had been published until April 2019, and conducted a meta-analysis for pooled incidence. The integrative search was undertaken in accordance with the Preferred Reporting Items for Systematic Reviews and Meta-Analyses (PRISMA) guidelines, by means of the term "alcoholic cirrhosis" AND "hepatoma" AND "incidence"; in the Airiti Library, the corresponding Chinese terms were applied for searching. The articles compatible with the inclusion criteria were retrieved, and literature published in English or Chinese was enrolled. Case reports and series, letters to the editor, in vitro studies, and studies on experimental models were excluded.

Three independent authors (T.H. Li, Y.L. Tsai, C.F. Hsu, and Y.Y. Yang) screened the abstracts and reviewed the full text adhering to the preset protocols; for disparities in literature appraisal, other authors discussed to achieve consensus. We extracted the author or study name, time of publication, country, number of participants, and incidence of HCC; for the data presented as cumulative incidence, the annual incidence rate was calculated by the formula as previously mentioned [12]. The potential publication bias was assessed by fail-safe $\mathrm{N}$, which presumed the estimated number of studies required to reduce the effect size to a nonsignificant level.

2.4. Statistical Analysis. The incidence rate of primary HCC among the study subjects was calculated. In the part of direct comparison between the groups with or without propranolol, we conducted a 1:2 pair-matched case-control cohort by means of nearest-neighbor PSM, which was adjusted by age, sex, Child-Pugh score, cirrhosis duration, and comorbidity after estimating the probability by logistic regression. Student's $t$-test and a chi-squared test were applied for continuous and categorical variables, respectively. The KaplanMeier method with log-rank test was utilized for comparison of the risks.

The difference of annual incidence between our study and the pooled incidence being deduced from previous literature was assessed using a $P$ value by the exact binomial test; 
TABLE 1: Baseline demographic data of subjects with alcoholic cirrhosis.

\begin{tabular}{|c|c|c|c|}
\hline Variables & Patients with propranolol use, $N=23(\%)$ & Patients without propranolol use (number), $N=46(\%)$ & $P$ \\
\hline Male & $20(90.0)$ & $43(93.4)$ & 0.743 \\
\hline $\operatorname{Age}^{\mathrm{a}}$ & $52.5 \pm 9.1$ & $54.3 \pm 12.0$ & 0.858 \\
\hline \multicolumn{4}{|l|}{ Superimposed etiology } \\
\hline $\mathrm{HBV}$ & $6(27.2)$ & $17(36.9)$ & 0.367 \\
\hline $\mathrm{HCV}$ & $1(4.3)$ & $6(13.0)$ & 0.365 \\
\hline Autoimmune hepatitis & 0 & $2(4.3)$ & 0.31 \\
\hline Other CLD & 0 & 0 & N/A \\
\hline Child-Pugh score $^{a}$ & $7.5 \pm 1.5$ & $7.3 \pm 1.5$ & 0.575 \\
\hline \multicolumn{4}{|l|}{ Comorbidity } \\
\hline IHD & $1(4.3)$ & 0 & 0.31 \\
\hline Hypertension & $5(22.7)$ & $11(23.9)$ & 0.547 \\
\hline COPD & $1(4.3)$ & $1(2.2)$ & 0.365 \\
\hline $\mathrm{DM}$ & $5(22.7)$ & $18(39.1)$ & 0.468 \\
\hline $\mathrm{TB}$ & 0 & $1(2.2)$ & 0.484 \\
\hline Hyperlipidemia & 0 & 0 & N/A \\
\hline ESRD & 0 & 0 & N/A \\
\hline Cirrhosis duration (months) ${ }^{\mathrm{a}}$ & $64.8 \pm 17.3$ & $63.8 \pm 25.0$ & 0.974 \\
\hline Incidence of HCC & $1(4.3)$ & $11(23.9)$ & 0.043 \\
\hline
\end{tabular}

CLD: chronic liver disease; COPD: chronic obstructive pulmonary disease; DM: diabetes mellitus; ESRD: end-stage renal disease; HBV: hepatitis B virus; HCV: hepatitis C virus; HCC: hepatocellular carcinoma; IHD: ischemic heart disease; N/A: not available; TB: tuberculosis. ${ }^{\mathrm{a}}$ Mean \pm standard deviation.

$P$ values adhered to two-sided tests, and those $<0.05$ were considered significant. With regard to the meta-analysis of annual incidence, the effect size was derived with a random-effects model. The heterogeneity between studies was examined via Cochran's $Q$ test, in which $P<0.05$ suggested significant heterogeneity. The $I$-square index was utilized for quantification of heterogeneity. Data analyses were performed by means of Statistical Product and Service Solutions V.26 (SPSS, IBM, Armonk, New York, USA) and Comprehensive Meta-Analysis V.3 (Biostat, Englewood, New Jersey, USA).

\section{Results}

3.1. Patient Characteristics and Annual Incidence Rate. For the propranolol group, a total of 369 hospitalized patients who had been admitted with the diagnosis of AC and a concurrent prescription of propranolol were identified, and we ruled out those who met the exclusion criteria: 157 subjects with irregular use or discontinuation of propranolol, $77 \mathrm{sub}-$ jects with insufficient medical records, 29 with previous HCC history, 18 with a history of liver transplantation or hepatobiliary surgery, 17 with propranolol use before year 2006, 11 with malignancy other than HCC, five with misdiagnosis of $\mathrm{AC}$, and one with documented intolerance of propranolol; among the remaining 54 subjects, we included those with newly diagnosed HCC more than six months after enrolment or with regular follow-up for three years; consequently, 23 subjects were enrolled.

For the nonpropranolol group, $677 \mathrm{AC}$ patients with admission were screened; after exclusion by the aforementioned criteria, a 1:2 pair-matched PSM was embarked on and yielded 46 subjects. The demographic data of the enrolled subjects is shown in Table 1, exhibiting no significant difference of the baseline variables between the two groups after PSM.

In our three-year observation, one male patient in the propranolol group was diagnosed as HCC in the $35^{\text {th }}$ month; comparatively, eleven patients in the nonpropranolol group were diagnosed as HCC during the $8^{\text {th }}$ to $36^{\text {th }}$ month, and the proportion of HCC incidence was significantly higher than that in the propranolol group $(P=0.043)$. In addition, the Kaplan-Meier analysis also disclosed a significantly higher cumulative incidence of HCC in the nonpropranolol group $(P=0.046$, Figure 1). Neither incidence of other malignancy nor mortality was found during the follow-up period.

3.2. Comparison to the Pooled Annual Incidence Rate of HCC in AC. A total of 1,949 publications were identified from PubMed, EMBASE, CENTRAL, and Airiti Library, with 596 duplications between these databases. After title and abstract reading, 1,137 citations were considered as irrelevant studies, 154 citations were review articles, and 30 citations were case reports or series. Thirty-two articles were reviewed by full text after the exclusion, and finally, eight studies were included in the present meta-analysis $[4,5,13-18]$. The flow chart is listed in Figure 2.

The eight studies covering 10,361 AC patients contained five European and three Asian works, and the reported annual incidence ranged from 0.4 to $5.6 \%$. The pooled annual incidence in the meta-analysis was $2.41 \%$ with high heterogeneity $\left(I^{2}=99.8 \%, P<0.001\right.$, Figure 3$)$. No further metaregression was performed because of the relatively few studies [19]. Publication bias was examined 


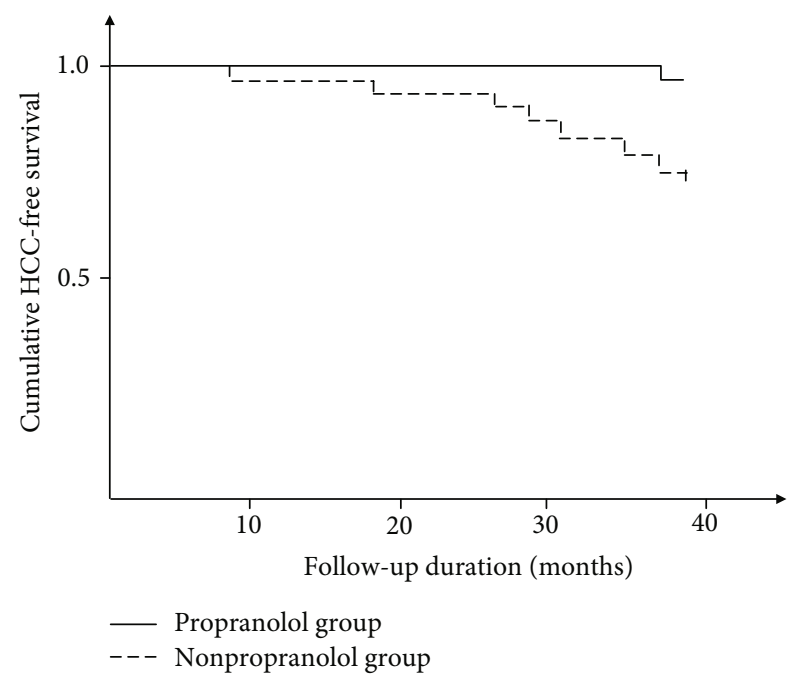

Figure 1: Kaplan-Meier curves for hepatocellular carcinoma-free survival in the propranolol group versus the nonpropranolol control group.

by means of Rosenthal's fail-safe number, which was 5,135 , indicating low publication bias.

The annual incidence in our present propranolol group was $1.45 \%$, which was significantly lower than the general pooled annual incidence garnered from the meta-analysis ( $1.45 \%$ vs. $2.41 \%$, respectively, $P=0.04)$. Namely, propranolol treatment in AC patients demonstrated reduction in the general annual incidence rate of HCC during the three-year follow-up.

\section{Discussion}

In the present study, propranolol treatment was associated with lower incidence of HCC in the AC group compared to the nonpropranolol group; moreover, we performed the meta-analysis of HCC incidence in AC patients to obtain more explicit data and further examined the effects of longterm propranolol use on HCC incidence. Herein, significant reduction of HCC incidence was embodied in our research.

Beta-adrenergic signaling plays a role in tumorigenesis and angiogenesis, and propranolol has manifested antitumor effects in various tumors [8-11, 20, 21]; as for in vitro studies of HCC, propranolol showed inhibition of proliferation and promotion of apoptosis in hepatoma cell lines [22]. However, data concerning the effects of propranolol on HCC incidence in cirrhotic patients obtained from clinical observational research remained controversial; some studies reported that the likelihood of developing HCC was lower in propranolol-treated patients than in those not treated, but other works indicated no significant difference in HCC incidence and survival $[23,24]$. Recently, one work aimed at the patients with cirrhosis on the waiting list for liver transplant yielded a protective effect for HCC, among whom 18 patients were attributed to alcohol abuse; however, these alcoholic subjects were not analyzed individually [25]. Actually, the aforementioned studies enrolled cirrhotic patients regardless of etiologies and did not con- duct subgroup analysis according to etiology, thus lacking data specific to AC patients.

Our work suggested that propranolol lowered the incidence of HCC in AC, of which the pathogeneses were mainly on the hepatic alcohol-mediated carcinogenesis resulting from the detrimental entities of acetaldehyde and the dysregulation of methylation, as well as immune alteration resulting from chronic inflammation [26]; these mechanisms were distinct from other etiologies such as viral hepatitis, and the antitumor effects might differ. In previous literature, propranolol had been mentioned with regard to counteractive properties to acetaldehyde [27, 28], modification of aberrant methylation pertinent to tumorigenesis [29], and anti-inflammatory roles in cirrhotic circumstances [30]; thus, the preventive effects on the HCC incidence of propranolol were theoretically grounded.

Despite the substantial proportion and enormous disease burden of AC, less research attention has been paid to it than to other etiologies such as viral hepatitis and nonalcoholic fatty liver disease [31]. Except for the well-known prognostic benefits of NSBB including propranolol for cirrhotic patients with portal hypertension, in patients with $\mathrm{AC}$ and alcoholic cardiomyopathy or congestive heart failure, NSBB improves quality of life, hemodynamics, and ejection fraction and reduces the severity of pulmonary hypertension $[32,33]$. In addition, NSBB has also been reported to prevent hepatosteatosis and attenuate progression in animal models of alcoholic liver diseases (ALDs) [34]. In combination with the antitumorigenesis results in the present study, NSBB such as propranolol provides multifarious benefits and may be a crucial treatment in patients with AC.

In addition to NSBB, the simultaneous prescription for the comorbidities such as DM and cardiovascular diseases in AC may influence the incidence of HCC. For example, metformin has been reported to reduce the risk of HCC in patients with DM [35]; notably, it is also associated with tumor aggressiveness and drug resistance in advanced HCC $[36,37]$. Besides, long-term aspirin use lowers the risk of HCC in the general population [38]; interestingly, lack of significant chemopreventive effect in cirrhotic patients has been proposed [39]. In general practice, the effects of these medications on HCC entail more studies to be unraveled.

As stated above, patients with cirrhosis carry a high risk of HCC. HCC screening in cirrhotic patients is an important issue in public health; the screening has been authenticated to decrease disease burden, reduce HCC rate, and improve survival conforming to standard practice [40-42]. In the view of the cost-effectiveness benefit, an annual incidence of HCC of $1.5 \%$ was regarded as the threshold of HCC screening [2]; although the annual incidence rates in $\mathrm{AC}$ are more than $1.5 \%$ in the majority of the literature, the result of $0.45 \%$ in a Danish national study puts the issue of HCC screening in AC patients in a dilemma $[4,43]$. Hence, we managed to explore a more accurate incidence rate by means of a metaanalysis, and the data suggested that the current HCC screening policies could be applied to AC patients.

Even when we attempted to include as much research as possible into our meta-analysis, the majority of the published literature was carried out in Western countries; therefore, 


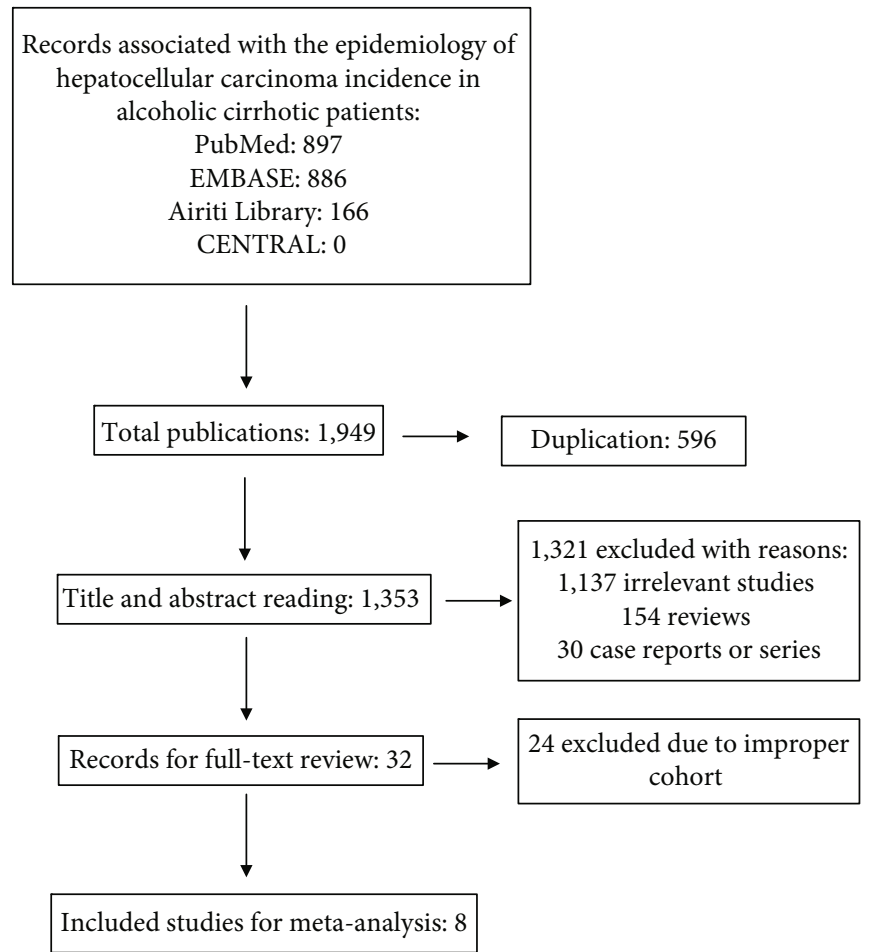

FIGURE 2: Flow chart of study inclusion for meta-analysis.

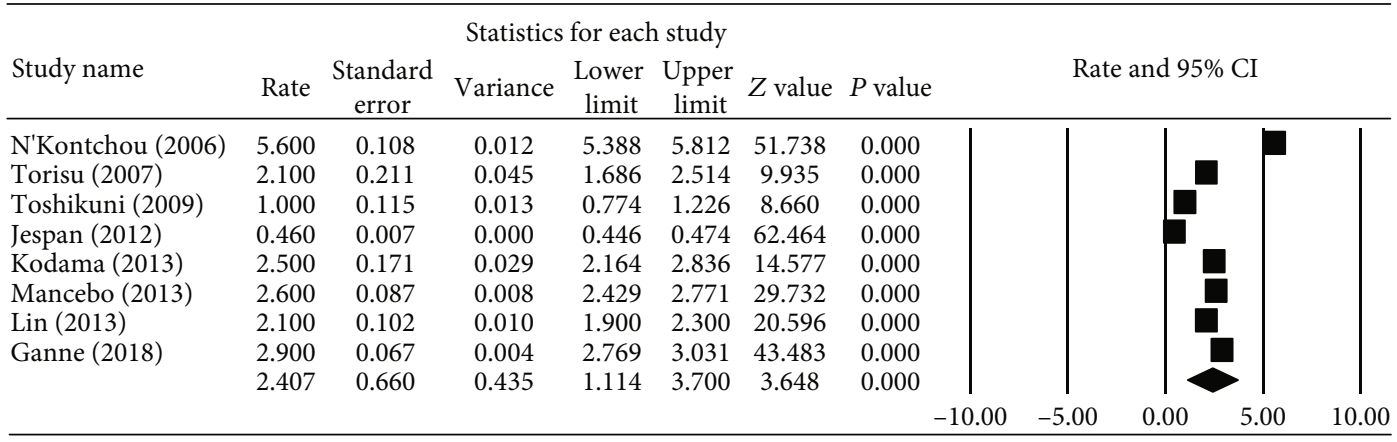

FIgURE 3: Forest plot of annual incidence rate of hepatocellular carcinoma in patients with alcoholic cirrhosis. The numbers shown in the picture were the percentage of annual incidence.

ethnical disparities might exist in our comparison. The only study undertaken in a Chinese population highlighted the superimposition of alcoholism on HBV-related HCC patients rather than on pure AC patients [17]. In addition, these studies did not analyze the incidence in terms of different severities, while the subjects in our study demanded hospitalization, and might result in more vulnerability to HCC compared to the general AC patients.

In spite of meticulous study design, our retrospective study has several limitations. First, the case number is comparatively few and thus may misestimate the accuracy of the incidence. In real-world practice, the adherence of medical instruction and the retention rate of observational study in patients with ALD are relatively low [44]; actually, concerning AC, the case number in our study is not less compared with the previous literature [23-25]. Second, although we applied PSM to reconcile the baseline bias, some information such as comorbidities was obtained retrospectively on the basis of medical records; therefore, we might have underestimated the proportion of the comorbidities. Also, the benchmark for the indirect comparison in the present study was the meta-analytic pooled annual incidence from the previous literature, among which part of the subjects might be treated with propranolol, which thus might offset the difference; however, even then, our data presented the effects of propranolol. Future works to elucidate the antitumorigenesis relationship and mechanism are warranted.

\section{Conclusions}

To conclude, we demonstrated the reduction of HCC incidence in patients with AC by propranolol; furthermore, we 
performed a meta-analysis of the annual incidence of HCC in AC. However, more epidemiologic data and larger-scale prospective cohort studies should be undertaken to ascertain the effects.

\section{Data Availability}

The data used to support the findings of this study are available from the corresponding author upon request.

\section{Conflicts of Interest}

All the authors declare that there are no conflicts of interest.

\section{Acknowledgments}

This work was supported by grants MOST-106-2511-S-010001-MY3 and MOST-108-2314-B-075-050-MY3 from the National Science Council.

\section{References}

[1] J. A. Flemming, J. D. Yang, E. Vittinghoff, W. R. Kim, and N. A. Terrault, "Risk prediction of hepatocellular carcinoma in patients with cirrhosis: the ADRESS-HCC risk model," Cancer, vol. 120, no. 22, pp. 3485-3493, 2014.

[2] J. Bruix, M. Sherman, and American Association for the Study of Liver Diseases, "Management of hepatocellular carcinoma: an update," Hepatology, vol. 53, no. 3, pp. 1020-1022, 2011.

[3] Global Burden of Disease Liver Cancer Collaboration, T. Akinyemiju, S. Abera et al., "The burden of primary liver cancer and underlying etiologies from 1990 to 2015 at the global, regional, and national Level," JAMA Oncology, vol. 3, no. 12, pp. 1683-1691, 2017.

[4] P. Jepsen, P. Ott, P. K. Andersen, H. T. Sørensen, and H. Vilstrup, "Risk for hepatocellular carcinoma in patients with alcoholic cirrhosis: a Danish nationwide cohort study," Annals of Internal Medicine, vol. 156, no. 12, pp. 841-7, W295, 2012.

[5] G. N'Kontchou, J. Paries, M. T. T. Htar et al., "Risk factors for hepatocellular carcinoma in patients with alcoholic or viral C cirrhosis," Clinical Gastroenterology and Hepatology, vol. 4, no. 8, pp. 1062-1068, 2006.

[6] K. T. Suk, M. Y. Kim, D. H. Park et al., "Effect of propranolol on portal pressure and systemic hemodynamics in patients with liver cirrhosis and portal hypertension: a prospective study," Gut and Liver, vol. 1, no. 2, pp. 159-164, 2007.

[7] C. Villanueva, A. Albillos, J. Genescà et al., " $\beta$ blockers to prevent decompensation of cirrhosis in patients with clinically significant portal hypertension (PREDESCI): a randomised, double-blind, placebo-controlled, multicentre trial," Lancet, vol. 393, no. 10181, pp. 1597-1608, 2019.

[8] L. Jean Wrobel, L. Bod, R. Lengagne, M. Kato, A. PrévostBlondel, and F. A. Le Gal, "Propranolol induces a favourable shift of anti-tumor immunity in a murine spontaneous model of melanoma," Oncotarget, vol. 7, no. 47, pp. 77825-77837, 2016.

[9] S. Ashrafi, R. Shapouri, A. Shirkhani, and M. Mahdavi, "Antitumor effects of propranolol: adjuvant activity on a transplanted murine breast cancer model," Biomedicine \& Pharmacotherapy, vol. 104, pp. 45-51, 2018.
[10] X. Liao, X. Che, W. Zhao, D. Zhang, T. Bi, and G. Wang, "The $\beta$-adrenoceptor antagonist, propranolol, induces human gastric cancer cell apoptosis and cell cycle arrest via inhibiting nuclear factor $\kappa \mathrm{B}$ signaling," Oncology Reports, vol. 24, no. 6, pp. 1669-1676, 2010.

[11] D. Zhang, Q. Yong Ma, H.-T. Hu, and M. Zhang, “ $\beta 2$-adrenergic antagonists suppress pancreatic cancer cell invasion by inhibiting CREB, NF- $\kappa \mathrm{B}$ and AP-1," Cancer Biology \& Ther$a p y$, vol. 10, no. 1, pp. 19-29, 2010.

[12] S. Greenland and K. J. Rothman, Measures of occurrence. In: Rothman, K.J. and Greenland, S. Modern Epidemiology, Lippincott Williams \& Wilkins, Philadelphia, 2008.

[13] Y. Torisu, K. Ikeda, M. Kobayashi et al., "Diabetes mellitus increases the risk of hepatocarcinogenesis in patients with alcoholic cirrhosis: a preliminary report," Hepatology Research, vol. 37, no. 7, pp. 517-523, 2007.

[14] N. Toshikuni, A. Izumi, K. Nishino et al., "Comparison of outcomes between patients with alcoholic cirrhosis and those with hepatitis C virus-related cirrhosis," Journal of Gastroenterology and Hepatology, vol. 24, no. 7, pp. 1276-1283, 2009.

[15] K. Kodama, K. Tokushige, E. Hashimoto, M. Taniai, and K. Shiratori, "Hepatic and extrahepatic malignancies in cirrhosis caused by nonalcoholic steatohepatitis and alcoholic liver disease," Alcoholism: Clinical and Experimental Research, vol. 37, suppl. 1, pp. E247-E252, 2013.

[16] A. Mancebo, M. L. González-Diéguez, V. Cadahía et al., "Annual incidence of hepatocellular carcinoma among patients with alcoholic cirrhosis and identification of risk groups," Clinical Gastroenterology and Hepatology, vol. 11, no. 1, pp. 95-101, 2013.

[17] C. W. Lin, C. C. Lin, L. R. Mo et al., "Heavy alcohol consumption increases the incidence of hepatocellular carcinoma in hepatitis B virus-related cirrhosis," Journal of Hepatology, vol. 58, no. 4, pp. 730-735, 2013.

[18] N. Ganne-Carrié, C. Chaffaut, V. Bourcier et al., "Estimate of hepatocellular carcinoma incidence in patients with alcoholic cirrhosis," Journal of Hepatology, vol. 69, no. 6, pp. 1274$1283,2018$.

[19] C. H. Schmid, J. Lau, M. W. McIntosh, and J. C. Cappelleri, "An empirical study of the effect of the control rate as a predictor of treatment efficacy in meta-analysis of clinical trials," Statistics in Medicine, vol. 17, no. 17, pp. 1923-1942, 1998.

[20] S. W. Cole and A. K. Sood, "Molecular pathways: betaadrenergic signaling in cancer," Clinical Cancer Research, vol. 18, no. 5, pp. 1201-1206, 2012.

[21] J. K. Wolter, N. E. Wolter, A. Blanch et al., "Anti-tumor activity of the beta-adrenergic receptor antagonist propranolol in neuroblastoma," Oncotarget, vol. 5, no. 1, pp. 161-172, 2014.

[22] F. Wang, H. Liu, F. Wang et al., "Propranolol suppresses the proliferation and induces the apoptosis of liver cancer cells," Molecular Medicine Reports, vol. 17, no. 4, pp. 5213-5221, 2018.

[23] H. Patel, A. Kumar, and N. Shah, "Role of non-selective beta blockers in hepatocellular carcinoma: an analysis in patients with cirrhosis and portal hypertension," North American Journal of Medicine \& Science, vol. 88, no. 33, pp. 105-108, 2015.

[24] T. W. Kim, H. J. Kim, C. U. Chon et al., "Is there any vindication for low dose nonselective $\beta$-blocker medication in patients with liver cirrhosis?," Clinical and Molecular Hepatology, vol. 18, no. 2, pp. 203-212, 2012. 
[25] N. Suna, D. Özer Etik, S. Öcal, and H. Selçuk, "Effect of propranolol treatment on the incidence of hepatocellular carcinoma in patients waiting for liver transplant with cirrhosis: a retrospective, surveillance study in a tertiary center," Experimental and Clinical Transplantation, vol. 17, no. 5, pp. 632637, 2019.

[26] H. K. Seitz and F. Stickel, "Molecular mechanisms of alcoholmediated carcinogenesis," Nature Reviews Cancer, vol. 7, no. 8, pp. 599-612, 2007.

[27] R. T. Rappolt, G. Gay, D. S. Inaba, N. Rappolt, and R. T. Rappolt, "Use of inderal (Propranolol-Ayerst) in I-a (early stimulative) and I-b (advanced stimulative) classification of cocaine and other sympathomimetic reactions," Clinical Toxicology, vol. 13, no. 2, pp. 325-332, 1978.

[28] H. Jänkälä, C. J. P. Eriksson, N. E. Petersen, M. Härkönen, and T. Mäki, "Role of acetaldehyde in the induction of heart left ventricular atrial natriuretic peptide gene expression in rats," Alcohol and Alcoholism, vol. 35, no. 4, pp. 331-335, 2000.

[29] E. F. Mong, K. M. Akat, J. Canfield et al., "Modulation of LIN28B/Let-7 signaling by propranolol contributes to infantile hemangioma involution," Arteriosclerosis, Thrombosis, and Vascular Biology, vol. 38, no. 6, pp. 1321-1332, 2018.

[30] A. Brito-Azevedo, R. M. Perez, H. S. Coelho et al., "The antiinflammatory role of propranolol in cirrhosis: preventing the inflammatory exhaustion?," Journal of Hepatology, vol. 66, no. 1, pp. 240-241, 2017.

[31] N. Ndugga, T. G. Lightbourne, K. Javaherian et al., "Disparities between research attention and burden in liver diseases: implications on uneven advances in pharmacological therapies in Europe and the USA," BMJ Open, vol. 7, no. 3, article e013620, 2017.

[32] A. G. Evdokimova, A. V. Tomova, O. I. Tereshchenko, L. V. Zhukolenko, and V. V. Evdokimov, "Clinical efficacy of the receptor beta and alpha blockers - carvedilol and ornithineaspartate in patients with CHD and CHF combined with alcoholic liver disease," Experimental \& Clinical Gastroenterology, vol. 6, pp. 42-47, 2016.

[33] A. Amor-Salamanca, G. Guzzo-Merello, E. González-López et al., "Impacto pronóstico y factores predictores de la recuperación de la fracción de eyección en pacientes con miocardiopatía dilatada alcohólica," Revista Española de Cardiología, vol. 71, no. 8, pp. 612-619, 2018.

[34] J. Liu, I. Takase, A. Hakucho, N. Okamura, and T. Fujimiya, "Carvedilol attenuates the progression of alcohol fatty liver disease in rats," Alcoholism Clinical and Experimental Research, vol. 36, no. 9, pp. 1587-1599, 2012.

[35] V. Cunha, H. P. Cotrim, R. Rocha, K. Carvalho, and L. LinsKusterer, "Metformin in the prevention of hepatocellular carcinoma in diabetic patients: a systematic review," Annals of Hepatology, 2019.

[36] A. Casadei Gardini, G. Marisi, E. Scarpi et al., "Effects of metformin on clinical outcome in diabetic patients with advanced HCC receiving sorafenib," Expert Opinion on Pharmacotherapy, vol. 16, no. 18, pp. 2719-2725, 2015.

[37] A. Casadei Gardini, L. Faloppi, S. de Matteis et al., "Metformin and insulin impact on clinical outcome in patients with advanced hepatocellular carcinoma receiving sorafenib: validation study and biological rationale," European Journal of Cancer, vol. 86, pp. 106-114, 2017.

[38] T. G. Simon, Y. Ma, J. F. Ludvigsson et al., "Association between aspirin use and risk of hepatocellular carcinoma," JAMA Oncology, vol. 4, no. 12, pp. 1683-1690, 2018.
[39] I. C. Hwang, J. Chang, K. Kim, and S. M. Park, “Aspirin use and risk of hepatocellular carcinoma in a national cohort study of Korean adults," Scientific Reports, vol. 8, no. 1, p. 4968, 2018.

[40] A. G. Singal, S. Mittal, O. A. Yerokun et al., "Hepatocellular carcinoma screening associated with early tumor detection and improved survival among patients with cirrhosis in the US," American Journal of Medicine, vol. 130, no. 9, pp. 10991106.e1, 2017.

[41] B. H. Zhang, B. H. Yang, and Z. Y. Tang, "Randomized controlled trial of screening for hepatocellular carcinoma," Journal of Cancer Research and Clinical Oncology, vol. 130, no. 7, pp. 417-422, 2004.

[42] N. Jiar, J. L. Slama, and N. Ganne-Carrié, "Screening of hepatocellular carcinoma in patients with uncomplicated cirrhosis in real life: practices survey of general practionners from three towns of Seine-Saint-Denis (north-east suburb of Paris)," Bulletin du Cancer, vol. 104, no. 7-8, pp. 618-624, 2017.

[43] J. Wakim-Fleming and R. Lopez, "Danish patients with alcoholic cirrhosis have a low risk of hepatocellular carcinoma thus raising questions about the utility of screening," EvidenceBased Medicine, vol. 18, no. 4, pp. 155-156, 2013.

[44] M. Comerford, S. Lourens, S. Liangpunsakul et al., "Challenges in patient enrollment and retention in clinical studies for alcoholic hepatitis: experience of the TREAT consortium," Alcoholism: Clinical and Experimental Research, vol. 41, no. 12, pp. 2000-2006, 2017. 